Editorial

\title{
Seventh International Conference on the Foundations of Information Science (FIS 2017): Structure, Dynamics, Complexity in the Informational Nature ${ }^{\dagger}$
}

\author{
Marcin J. Schroeder 1,*, Pedro C. Marijuán ${ }^{2, *}$ and Gordana Dodig-Crnkovic ${ }^{3, *}$ \\ 1 Akita International University, 193-2 Okutsubakidai, Yuwa-machi, 010-1211 Akita, Japan \\ 2 Instituto Aragonés de Ciencias de la Salud (I+CS), 50009 Zaragoza, Spain \\ 3 Chalmers University of Technology, Gothenburg, 40482, Sweden \\ * Correspondence: mjs@aiu.ac.jp (M.J.S.); pcmarijuan.iacs@aragon.es (P.C.M.); \\ gordana.dodig-crnkovic@chalmers.se (G.D.-C.) \\ + Presented at the IS4SI 2017 Summit DIGITALISATION FOR A SUSTAINABLE SOCIETY, Gothenburg, \\ Sweden, 12-16 June 2017.
}

Published: 3 August 2017

Information Science evolved in the last few decades from peripheral studies of other disciplines which used the concept of information with a more or less common-sense meaning, to a fully-fledged discipline with a growing toolkit of concepts and methods of inquiry. This does not mean that the concept of information acquired a unique definition across research and application fields or that there is a consensus regarding its theoretical description. However no nontrivial concept of science or philosophy has such unchallenged, uniform formulation.

Foundation of Information Science initiative which was started almost 25 years ago and acquired increasingly organized form through the following decades remains faithful to the original ideas summarized at its early stage by Pedro Marijuán [1]: “[...] perhaps the nucleus of the FIS initiative, would be the quest for a unifying approach capable of introducing a new conceptual order into the contemporary mosaic of disparaging acceptations around the term information. As Michael Conrad and this author argued in the first FIS venue [2,3], rather than discussing about a unitary meaning for the term, understanding information from the FIS premises appears as the intellectual adventure of developing a 'vertical' science connecting, so to speak, the different scales of informational processes - reminding physics itself, which from a pre-Galilean particularized single term evolved towards a vertical science connecting the previously separated 'celestial', 'sublunar', and 'terrestrial' physical occurrences".

The history of FIS was punctuated by its conferences with very diverse and extensive programs:

- 1994-First Conference on Foundations of Information Science: From computers and quantum physics to cells, nervous systems, and societies. Madrid, Spain, July 11-15, 1994.

- 1996-Second Conference on Foundations of Information Science: The Quest for a Unified Theory of Information. Vienna, Austria, June 11-15, 1996.

- 2005-Third Conference on Foundations of Information Science: Seeking an unifying field for science of information. Paris, France, 4-7 July 2005.

- 2010-Fourth International Conference on Foundations of Information Science: Towards a new Science of Information, Beijing, China, 21-24 August 2010.

- 2013-Fifth International Conference on Foundations of Information Science: Perspectives of Information in Global Education as a New Approach for the 21st Century. Moscow, Russia, 21-24 May 2013.

- 2015-The Sixth International Conference on Foundations of Information Science: Triangular relationship: Information-Reality-Cognition. Vienna, Austria, June 3-7, 2015. 
The Seventh International Conference on Foundations of Information Science: Structure, Dynamics, Complexity in the Informational Nature was a part of the International Society for the Study of Information (is4si) 2017 Summit at Gothenburg, Sweden held at Chalmers Institute of Technology June 12-16, 2017 had overarching theme: DIGITALISATION FOR A SUSTAINABLE SOCIETY: Embodied, Embedded, Networked, Empowered through Information, Computation \& Cognition.

Maturation of Information Science changed its role. At the beginning explanation of information was sought within the disciplines where the concept was used. In time it is the concept of information and its characteristics that serve as intellectual tools to solve problems of other disciplines. The expression "Informational Nature" is expressing the fact that nature, the subject of traditional scientific inquiry (in physics, chemistry, biology, etc.) requires for its study and understanding the concept of information and methods of Information Science.

As every other discipline, Information Science has its own challenges and the title of the Seventh International Conference on the Foundations of Information Science: Structure, Dynamics, Complexity in the Informational Nature points to great challenges for the intellectual inquiry, scientific exploration of reality or technological innovation. Our goal was to stimulate attempts to overcome these challenges. Moreover explanatory power of the concept of information in the variety of contexts and disciplines brings hope that the old dream of science to acquire a holistic view of reality can be achieved $[4,5]$. The search for such a unifying perspective can be considered an ultimate goal of Information Science.

We invited contributions to the conference which use the informational perspective to explore the issues associated with concepts of structure, dynamics and complexity encountered in nature and to resolve problems related to limitations and challenges associated with them. For instance, the problem of overcoming the limitations of complexity (one of the greatest challenges of present time) may find solution through nature inspired innovative forms of computation exemplifying dynamics of information. On the other hand, our increasing understanding of computation or dynamics of information may give us novel methods of exploration of nature. Reflection on this and other challenges was of great interest for the conference.

We did not set any limitations on the way the mutual relations between the key concepts of structure, dynamics and complexity in informational nature are studied, but we expected that the concept of information will serve well as a point of reference for the exchange of views between participants of the conference. This should not be a surprise that one of the most frequently invoked questions in the discussions held between presentations as well as within the discussion which we organized and scheduled as a culmination of the conference was "What is information?".

The contributions that follow this note are short versions of papers presented at the conference. We hope that they will grow into extensive studies published in the other venues documenting the Summit.

\section{References}

1. Marijuán, P.C. Foundations of Information Science: Selected papers from FIS 2002. Entropy 2003, 5, 214-219.

2. Conrad, M. Cross-scale information processing in evolution, development and intelligence. BioSystems 1996, 38, 97-109.

3. Marijuán, P.C. Foundations of Information Science: FIS 1994 Conference Proceedings. BioSystems 1996, 38, 87-96.

4. Schroeder, M.J. The Philosophy of Philosophies: Synthesis through Diversity, Editorial. Philosophies 2016, 1, 68-72.

5. Dodig-Crnkovic, G. Dynamics of Information as Natural Computation. Information 2011, 2, 460-477; doi:10.3390/info2030460. 